\title{
METODOLOGIA DE CLASSIFICAÇÃO DAS \\ COMPONENTES ANGULARES DE TEODOLITOS E \\ ESTAÇÕES TOTAIS EM LABORATÓRIO
}

\begin{abstract}
Methodology for classifying angular componens of theodolites and total stations in laboratory
\end{abstract}

\author{
MARIA MADALENA SANTOS DA SILVA ${ }^{1}$ \\ PEDRO LUIS FAGGION ${ }^{2}$ \\ LUIS AUGUSTO KOENIG VEIGA ${ }^{2}$ \\ ${ }^{1}$ Universidade Tecnológica Federal do Paraná \\ mariasantos@utfpr.edu.br \\ ${ }^{2}$ Universidade Federal do Paraná \\ faggion@ufpr.br, kngveiga@ufpr.br
}

\begin{abstract}
RESUMO
Este artigo trata do desenvolvimento de uma metodologia para classificação angular (vertical e horizontal) de teodolitos e estações totais. A metodologia baseou-se na norma brasileira (NBR 13.133) e internacional (ISO 17.123-3). Para atingir este objetivo diversas etapas foram desenvolvidas, inicialmente transformou-se a luneta de um teodolito óptico em um colimador. Também foram desenvolvidos seus filtros e iluminação, bem como a bancada suporte. Com a finalização desta etapa partiu-se para a montagem do laboratório e realização dos testes. Na classificação angular horizontal, foram comparados os testes realizados em base de campo com os de laboratório. Os dados obtidos foram aplicados em um programa que emite a classificação do equipamento de acordo com a NBR 13.133. E por fim foram realizados testes com colimadores comerciais e os resultados comparados com os obtidos anteriormente. Na classificação angular vertical foram comparados os dados obtidos no laboratório com os colimadores construídos e com os colimadores industriais. Todos os dados foram avaliados com os testes estatísticos Global e Data Snooping. Os resultados finais confirmaram a eficiência da metodologia desenvolvida na criação do laboratório de classificação angular horizontal e vertical, bem como o resultado satisfatório do colimador construído no laboratório e que apresentou baixo custo de desenvolvimento.
\end{abstract}


Palavras-chave: Classificação angular horizontal e vertical; colimadores; testes estatísticos.

\section{ABSTRACT}

This article is about the development of angular classification methodology (vertical and horizontal) of theodolites and total stations. The methodology was based on Brazilian (NBR 13.133) and International (ISO 17.123-3) rules. In order to accomplish that objective, several steps were developed; initially the telescope of an optical theodolite was turned into a collimator. Its filters and illumination were also developed, as well as its support. After this step, the assembling of a laboratory and the performance of tests began. At horizontal angular classification, field tests were compared with laboratory tests. Data obtained were applied into an arithmetic spreadsheet that issues the classification of the equipment according to NBR 13.133. And at last, tests were performed with industrial collimators which were compared to previous results. In the vertical angular classification, data obtained from laboratory with assembled collimators were compared to data of industrial collimators. All data were evaluated with Global and Data Snooping statistical tests. Final results confirmed the efficiency of the developed methodology to create a laboratory of horizontal and vertical angular classification at the UFPR, as well as the satisfactory result of the assembled collimator on laboratory that presented low development cost.

Keywords: horizontal and vertical angular classification; collimators; statistical tests.

\section{INTRODUÇÃO}

A aplicação de métodos geodésicos e topográficos na engenharia é ampla: locações de obras, determinações de desníveis, monitoramento de estruturas, são alguns exemplos destas aplicações. Porém, para garantir a qualidade e precisão requerida para execução destes trabalhos é importante que os instrumentos atendam a determinados critérios de precisão. Para tanto é essencial que periodicamente sejam realizadas calibrações, verificações, retificações (quando necessário) e classificação destes instrumentos.

A execução periódica destas atividades se justifica pelo uso contínuo e extensivo dos equipamentos no decorrer dos anos, longos períodos de armazenamento, transporte e procedimentos inadequados. Essas questões podem provocar nas peças um desgaste levando o equipamento a operar fora dos padrões estabelecidos originalmente.

No Brasil, a norma que estabelece valores de referência para classificação destes equipamentos é a NBR 13.133, publicada em 1994. Seu texto apresenta as exigências mínimas para a classificação de teodolitos de acordo com a precisão angular e estações totais em função da precisão angular e linear.

A NBR 13.133 estabelece no seu item 4.1.1.1 que a classificação dos 
teodolitos e estações totais, bem como outros instrumentos geodésicos e topográficos, só pode ser realizada por entidades oficiais e/ou Universidades, em bases apropriadas. Cabe salientar que a NBR 13.133 apresenta apenas os procedimentos a serem executados para a classificação da componente angular horizontal em campo.

A norma internacional que estabelece as diretrizes para procedimentos de testes com instrumentos ópticos é denominada ISO 17.123 - Optics and optical instruments - Field procedures for testing geodetic and surveying instruments Part 3: Theodolites, sendo sua primeira versão datada de 01/12/2001. A parte 3 da referida norma estabelece os procedimentos para os testes completos e testes simplificados de campo, para a componente horizontal e vertical separadamente.

Neste trabalho buscou-se desenvolver uma metodologia para a realização da classificação da componente angular de teodolitos e estações totais em laboratório, em ambiente com temperatura controlada, aplicando-se as orientações estabelecidas pela NBR 13.133 e ISO 17.123-3. Como um dos seus resultados tem-se a implantação de um laboratório específico para este fim.

\section{METODOLOGIA}

A metodologia para a classificação das componentes angulares (horizontal e vertical) de teodolitos e estações totais em laboratório é composta pelas seguintes etapas:

a. Análise dos instrumentos disponíveis para construção dos colimadores, desenvolvimento do projeto da bancada e projeto de distribuição dos colimadores;

b. Estudo e desenvolvimento da iluminação dos colimadores montados a partir de teodolitos, testes preliminares da viabilidade do uso de lunetas de teodolitos DF Vasconcelos, como colimadores e análise da influência de diferentes iluminações nas observações realizadas nos colimadores;

c. Testes na base horizontal do laboratório utilizando os colimadores construídos no laboratório e implantação da base vertical no laboratório conforme a ISO 17.123-3, utilizando os colimadores construídos;

d. Desenvolvimento da planilha eletrônica de cálculo e certificação do resultado da classificação horizontal conforme a ISO 17.123-3, desenvolvimento da planilha eletrônica de cálculo e certificação do resultado da classificação vertical conforme a ISO 17.123-3;

e. Instalação dos colimadores Leica na base do laboratório, teste na base do laboratório com os colimadores Leica;

f. Comparação entre os resultados das três bases (campo, laboratório com colimadores construídos e laboratório com colimadores Leica) e análise dos resultados obtidos. 


\subsection{Análise dos Instrumentos Disponíveis para Construção dos Colimadores, e do Projeto da Bancada.}

A classificação da componente angular em laboratório está baseada no uso de colimadores distribuídos tanto horizontalmente como também na vertical, os quais são utilizados como referência para a realização das pontarias. Inicialmente buscou-se soluções para a construção dos colimadores, de forma a apresentar uma alternativa funcional e de custo reduzido, se comparada com colimadores comerciais. Desta forma, se buscou opções satisfatórias com recursos disponíveis e adaptáveis, sendo a solução adotada a adaptação de lunetas de teodolitos.

Dentre os equipamentos disponíveis, mas que em contrapartida permitissem a solução desejada optou-se pelo teodolito DF Vasconcelos. Tais equipamentos estavam em desuso e apresentavam pequenas avarias na parte mecânica, como por exemplo, nas bases nivelantes. Mas suas lunetas, que são do tipo analáticas (possibilitam projetar um ponto no infinito), encontravam-se em bom estado de conservação. Para esta constatação foram realizadas observações e testes para verificar a inexistência de fungos nas lentes ou avarias no conjunto.

Com a adaptação de um sistema de iluminação e de um conjunto de filtros, foi possível converter a luneta em um colimador.

Em paralelo foi desenvolvido o projeto da bancada para a base horizontal de classificação, na qual foram fixados os colimadores para a classificação da componente angular horizontal. Esta bancada possui o lay-out de acordo com a figura 1. Foi organizado um arranjo com cinco colimadores, que materializam os ângulos horizontais aproximados de: $0^{\circ}, 90^{\circ}, 139^{\circ}, 180^{\circ}$ e $279^{\circ}$. No centro desta bancada existe um dispositivo de centragem forçada com altura regulável para a instalação do equipamento.

Figura 1 - Arranjo horizontal de colimadores sobre a bancada.

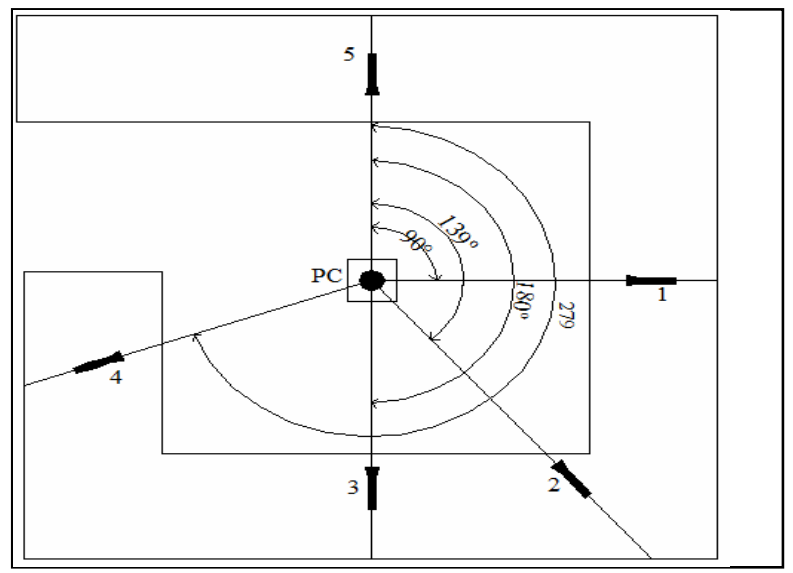

Bol. Ciênc. Geod., sec. Artigos, Curitiba, v. 16, nº 3, p.403-419, jul-set, 2010. 
A disposição dos colimadores no arranjo apresentado foi definida buscando-se colocar dois dos cinco colimadores em posições divergentes de $180^{\circ}$. Os demais colimadores foram colocados de forma estratégica na bancada, e sua funcionalidade baseia-se na repetibilidade de observações durante os testes.

$\mathrm{Na}$ seqüência do trabalho, foram selecionados os teodolitos que poderiam ser disponibilizados. Feita a seleção passou-se a realização da identificação das avarias e limpeza das lentes.

A base vertical foi elaborada com base nas indicações da ISO 17.123-3. Os colimadores desenvolvidos no laboratório foram fixados na parede do mesmo. Por questões estruturais do colimador, foi necessário o desenvolvimento de uma plataforma suporte para os colimadores, com dimensões e características necessárias para suportar de forma estável o sistema colimador. Os colimadores foram posicionados de acordo com os seguintes ângulos zenitais aproximados de: $54^{\circ} \mathrm{e}$ $66^{\circ}$ (posicionados acima da bancada da base horizontal), $90^{\circ}$ (sobre a bancada da base horizontal) e $116^{\circ}$ (abaixo da bancada da base horizontal), conforme mostra a figura 2. Os ângulos zenitais de $54^{\circ}$ e $66^{\circ}$ foram determinados pela limitação de observação através da ocular devido a sua inclinação.

Figura 2 - Base de classificação angular vertical

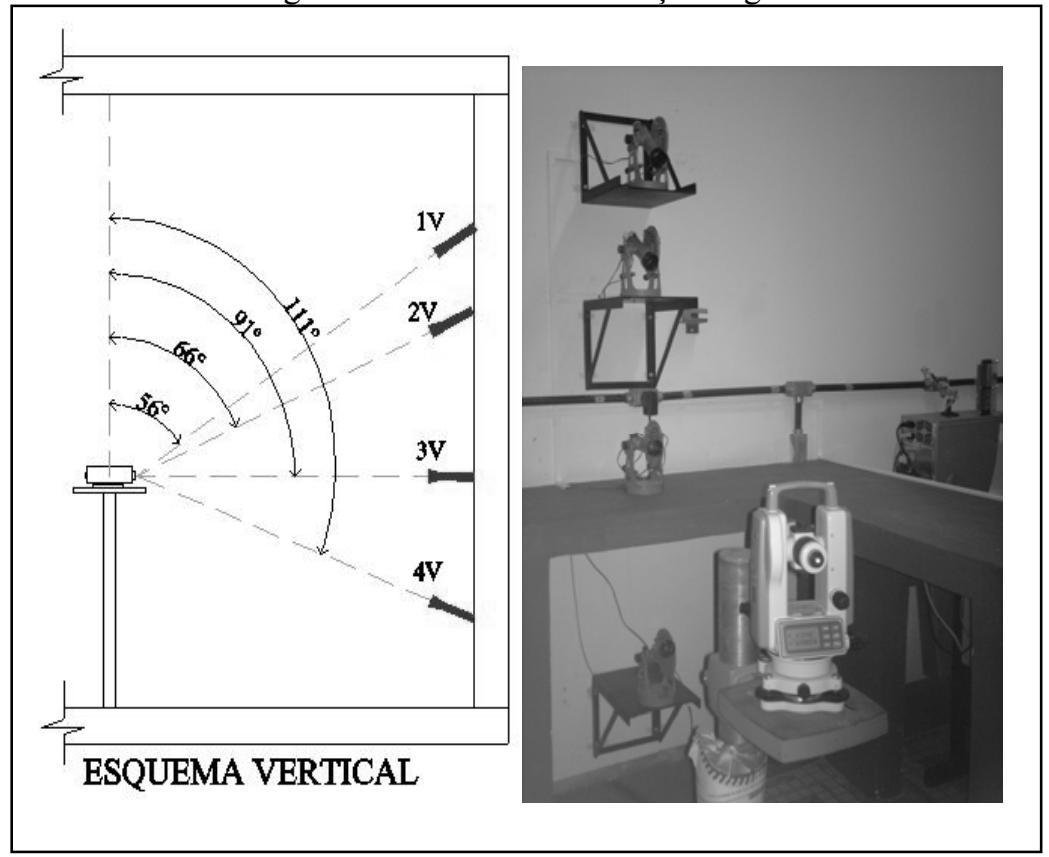

Bol. Ciênc. Geod., sec. Artigos, Curitiba, v. 16, n 3, p.403-419, jul-set, 2010. 


\subsection{Estudo e Desenvolvimento do Sistema de Iluminação dos Colimadores.}

Concluída as etapas de preparo das lunetas dos teodolitos e da bancada horizontal, iniciou-se o desenvolvimento do sistema de iluminação. Com este fim foram testados diversos tipos de iluminação, dentre eles a iluminação com lâmpada dicróica, iluminação com lâmpada incandescente e iluminação com LED. Nestes testes os diversos tipos de iluminação foram associados a diferentes filtros (com diferentes materiais e cores). Problemas encontrados durante os testes foram: intensidade da iluminação insuficiente, imagem turva dos fios de retículo, efeitos estes que provocavam cansaço na realização das medidas e aquecimento interno da luneta provocando efeito de reverberação na imagem dos fios de retículo. Por fim, após diversos testes, chegou-se a iluminação composta por LED (Light Emitting Diode) e filtro na cor azul. O LED é um diodo semicondutor que se energizado emite luz visível. Este dispositivo propicia uma luz pontual intensa e fria, o que resolve o problema do aquecimento e da intensidade de iluminação e o filtro azul proporciona conforto visual ao observador. Este conjunto de iluminação propicia a visualização nítida dos fios de retículo, bem como a colimação entre os fios de retículo do equipamento em processo de classificação e do colimador.

Uma constatação importante na comparação entre as diversas iluminações, é que a variação na intensidade de iluminação propiciou variação de focalização nos testes. Nos testes realizados entre a iluminação com lâmpadas do tipo dicróica e incandescente, utilizou-se um teodolito eletrônico T105 cuja precisão angular é de 5". Durante a realização dos testes observou-se uma discordância de \pm 11 " para a mesma pontaria, trocando-se apenas a fonte de iluminação no colimador. O mesmo efeito não ocorre com o $L E D$ por emitir um ponto de luz constante. $\mathrm{O}$ teste foi realizado da seguinte forma: focalizou-se o fio de retículo do colimador equipado com a lâmpada dicróica. Posteriormente foi substituída esta iluminação, pela iluminação com a lâmpada incandescente. Utilizando o LED não foram necessárias ajustes de focalização, devido à fonte contínua de iluminação.

\subsection{Avaliação da Base Horizontal e Vertical de Laboratório Utilizando os Colimadores Construídos}

Para avaliar a qualidade do colimador construído, foram realizados testes com sete equipamentos (estações totais e teodolitos) de marcas e modelos distintos. Isto porque a diversificação de fabricantes implica em variação do sistema óptico de suas lunetas.

Estes testes consistiram na instalação dos equipamentos na base de laboratório e realização de séries de observação. Adicionalmente, os mesmos equipamentos foram classificados na base de campo já existente de forma a permitir a comparação entre os resultados de laboratório e campo. Para estes testes utilizou-se um teodolito DF Vasconcelos, teodolito BD3 World marca Wild, teodolito T2 Wild, teodolito T100 Leica, teodolito T105 Leica, estação total TC 403L Leica e estação total TC 
2002 Leica.

Os testes realizados para verificar a adequação dos equipamentos a base foram realizados da seguinte forma: cada um dos equipamentos foi instalado no pilar central da base e foram realizadas as pontarias nos colimadores construídos (fios dos retículos) de forma a verificar se era possível a focalização nos mesmos. Todos os equipamentos testados corresponderam às expectativas e se adaptaram a base.

Para a avaliação dos resultados obtidos na classificação angular empregandose a base de laboratório, buscou-se comparar as duas metodologias, a de campo e a de laboratório. A base de campo da UFPR localiza-se na região próxima a pista de atletismo do campus Centro Politécnico e foi construída contendo um pilar central e mais quatro pilares dotados de sistema de centragem forçada, distantes do pilar central de aproximadamente $185 \mathrm{~m}$, seu desenvolvimento pode ser encontrado em MEDINA (1998). Esta base foi construída de acordo com as recomendações da NRB 13.133.

Para este experimento foi empregada a estação total TC403L, cuja precisão angular nominal é de \pm 10 ”, 4 alvos refletores, psicrômetro e guarda-sol. No início de cada série foi observada a temperatura ambiente, em média $26,5^{\circ} \mathrm{C}$. As observações foram realizadas na base de campo no período da tarde, a partir das 14 horas, havia presença de vento fraco, sendo possível a realização de boa pontaria nos alvos. Foram executadas 2 coletas de observações, cada uma com 3 séries completas (PD e PI) observando todos os pilares da base. O tempo necessário para a realização do teste totalizou 3 horas e 40 minutos. No laboratório a estação total TC403L foi instalada e nivelada no pilar central da base e foram observados 4 colimadores. Utilizou-se o mesmo número de alvos em laboratório e campo, para compatibilizar os resultados, de forma a não alterar a ponderação no ajustamento das observações, implementado na planilha de classificação eletrônica.

Em laboratório o sistema de ar-condicionado foi ligado com antecedência de 1 hora, para a adequação da temperatura do equipamento com a do ambiente, a qual foi mantida em $21^{\circ} \mathrm{C}$. Foram realizadas 3 séries completas (PD e PI) em quatro colimadores. O tempo para a realização do teste foi de 1 hora e 20 minutos.

Cabe salientar que as comparações entre as classificações realizadas em campo e laboratório foi realizada apenas para componente angular horizontal, visto que não é possível de realizar esta classificação da componente vertical em campo. Essa questão foi contornada da seguinte forma, os testes de classificação angular horizontal e vertical em laboratório foram feitos separadamente. Primeiramente foram executados os testes para a base angular horizontal, todos os dados foram registrados e avaliados na planilha eletrônica desenvolvida com esse fim.

Para a base angular vertical seguiram-se os procedimentos de teste completo indicados na ISO 17.123-3 adaptados ao laboratório e para as análises de classificação utilizaram-se os parâmetros de classificação angular horizontal de acordo com a tabela da NBR 13.133. Isto foi necessário, pois a ISO 17.123-3 sugere que os valores utilizados na classificação angular horizontal sejam utilizados na 
classificação angular vertical, pois ambos tratam-se de parâmetros angulares.

\subsection{Instalação e Teste dos Colimadores Leica na Base do Laboratório}

Em virtude dos resultados promissores dos testes realizados no laboratório a partir dos colimadores construídos com teodolitos, a pesquisa obteve um financiamento junto a Fundação Araucária de Apoio ao Desenvolvimento Científico e Tecnológico do Paraná, que propiciou a aquisição de um sistema colimador marca Leica. Este sistema é constituído por: 5 colimadores, sistema para fixação, uma caixa de distribuição, sistema de iluminação, parafusos, cabos e presilhas. A montagem do sistema se inicia pela acoplagem do sistema de iluminação aos colimadores, que possuem a aparência das lunetas do teodolito T105. O sistema de iluminação é similar ao desenvolvido em laboratório, possui um filtro (cor amarelo) e um ponto de iluminação e são encaixados por meio de rosca a parte posterior (onde seria a ocular) do colimador. A figura 3 apresenta o colimador após a finalização da montagem do sistema. A fixação dos colimadores sobre a bancada e nas paredes se dá por um sistema de placas de encaixe e disposto de forma similar ao implantado no sistema desenvolvido no laboratório.

Figura 3 - Colimador Leica montado sobre a base de classificação do laboratório ao conjunto.

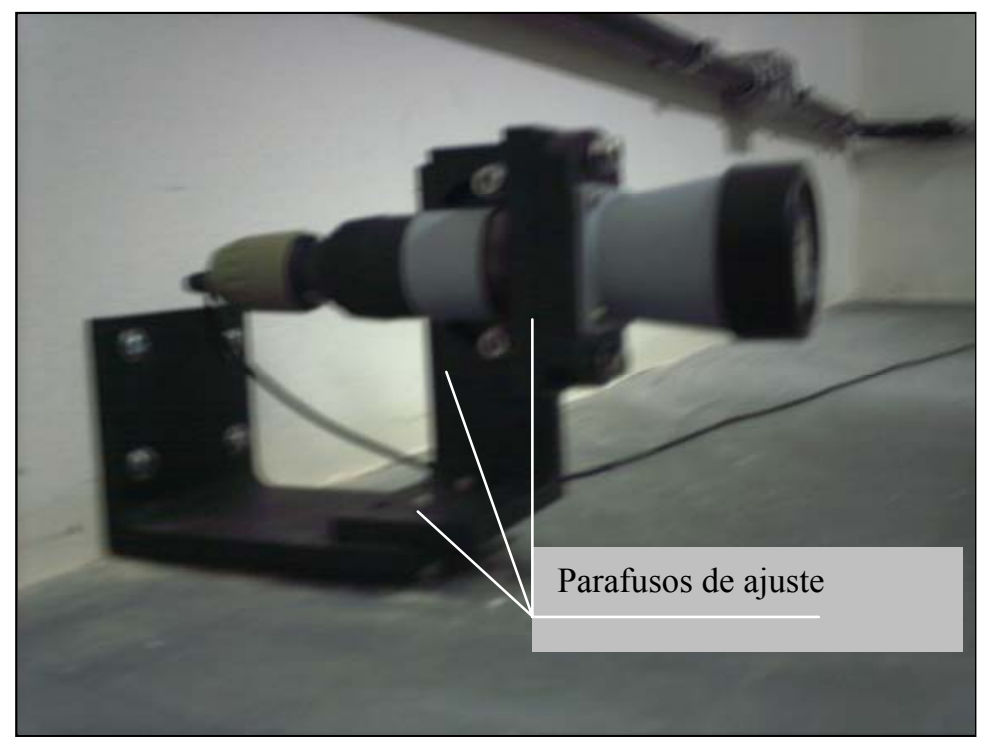

A base de classificação foi montada atendendo a um arranjo que contemple a parte horizontal e vertical da classificação angular, orientando cada colimador na 
direção do pilar central. A execução da orientação é uma etapa delicada, pois ajustes milimétricos devem ser feitos no sistema de encaixe do colimador através de parafusos de ajuste (figura 3). Os ajustes são realizados de forma a orientar o colimador e o equipamento posicionado no pilar da base em posição de colimação adequada. O sistema de iluminação é alimentado por energia elétrica, sendo utilizado um transformador de tensão de $6 \mathrm{~V}$.

Concluída a etapa de instalação e colimação do sistema colimador, passou-se a fase de testes. Nesta etapa do trabalho, foram testados os novos colimadores e realizada uma avaliação em relação aos resultados obtidos pelos colimadores construídos no laboratório. Para isso, foram realizados 5 testes para a classificação horizontal e 5 testes para a classificação vertical, sendo que duas vezes com o mesmo equipamento em cada classificação, para garantir a repetibilidade dos resultados. Os equipamentos testados foram teodolitos eletrônicos T100, T105 (duas vezes) e a estação total TC403 L.

O procedimento de execução é similar ao realizado nos colimadores construídos, descrito anteriormente. A climatização do ambiente deve acontecer com 30 minutos de antecedência à realização dos testes, para a adequação da temperatura dos equipamentos. $\mathrm{O}$ instrumento deve ser posicionado sobre o pilar central da base de classificação e na seqüência, inicia-se o procedimento de obtenção das observações. $\mathrm{O}$ operador em posição direta da luneta realiza a pontaria e então zera o equipamento no colimador 1 . A direção igual a $0^{\circ}$ deve ser anotado na planilha da classificação horizontal. Em seguida, prosseguem-se as observações em sentido horário e realizando as observações nas duas posições da luneta (PD e PI). Esta sequência deve ser seguida até finalizar as 3 séries completas de observações.

\subsection{Desenvolvimento da Planilha Eletrônica de Cálculo para Classificação Angular Horizontal e Vertical Conforme a ISO 17.123-3}

Para o processamento das observações de campo e laboratório, foi desenvolvida uma planilha eletrônica de cálculo, ou também denominada relatório de classificação angular. A planilha foi desenvolvida em ambiente Excel, de acordo com a formulação apresentada na NBR 13.133/1994 anexo C. A versão final desta planilha eletrônica contempla 5 colimadores, sendo campos de entrada obrigatórios o número de séries e conjuntos envolvidos no cálculo e o número de colimadores observados. Em laboratório são realizadas 4 séries completas de observações, compostas por 3 conjuntos cada uma das séries. Nesta planilha também foi implementada a avaliação estatística recomendada na ISO 17.123-3. No corpo da planilha o observador necessita entrar com os dados de observação, ou seja, os ângulos medidos em PD e PI.

O resultado da classificação angular é dado considerando a média angular, resíduos dos ângulos medidos em relação a média angular, resíduo angular total de cada série individualmente, desvio padrão de cada série individualmente, graus de 
liberdade de cada série, graus de liberdade total e desvio-padrão final. É em virtude do desvio-padrão final, que se classifica o equipamento em alta, média ou baixa precisão de acordo com o quadro 1, baseado na NBR13.133.

Quadro 1 - Classificação angular horizontal conforme NBR13.1
\begin{tabular}{|c|c|}
\hline Classes de equipamentos & $\begin{array}{c}\text { Desvio-padrão } \\
\text { Precisão angular }\end{array}$ \\
\hline 1 - Precisão baixa & $\leq \pm 30 "$ \\
\hline 2 - Precisão média & $\leq \pm 07$ " \\
\hline 3 - Precisão alta & $\leq \pm 02 "$ \\
\hline
\end{tabular}

FONTE: NBR 13.133 (1994)

A figura 4, apresenta os resultados obtidos no experimento realizado em laboratório, de acordo com os procedimentos da ISO 17.123-3 e tabela da NBR13.133, para o teodolito eletrônico T105, cuja precisão angular nominal é \pm 5 ".

Figura 4. - Resultado final obtido na classificação horizontal conforme ISO17.1233 - teste completo T105.

\begin{tabular}{|c|c|c|}
\hline \multicolumn{3}{|c|}{ CLASSIFICAÇÃO DO INSTRUMENTO (ÂNGULOS HORIZONTAIS)- TESTE COMPLETO } \\
\hline \multicolumn{3}{|c|}{ INSTRUMENTO DE MÉDIA PRECISÃO } \\
\hline Desv. Padräo série 1 (S1) (") & Desv. Padrăo série 3 (S3) ("') & Graus de liberdade \\
\hline 3,6514 & 4,6547 & pl i série (GLi) \\
\hline Desv. Padräo série 2 (S2) (") & Desv. Padrão série 4 (S4) ("') & 8 \\
\hline 3,9264 & 5,5527 & Graus de liberdade \\
\hline Desvio Padrăo Experimental S (") & Características do Equipamento & pl todas as séries (GL) \\
\hline 4,51 & Bom estado de conservaçăo & 32 \\
\hline \hline
\end{tabular}

A planilha de classificação encerra-se após a obtenção do desvio-padrão experimental $S$, que indica a classificação do equipamento. O passo seguinte realiza a análise estatística do grupo de observações. Conforme apresentado na norma ISO 17.123-3, existem alguns questionamentos acerca do desvio-padrão experimental calculado e do desvio-padrão fornecido pelo fabricante, e também com relação à homogeneidade da população de dados. Feitas as ponderações o resultado alcançado é explicitado, como no exemplo apresentado na figura 5.

A análise estatística procede de acordo com a recomendação da ISO 17.123-3. 
Figura 5 - Resultado do teste estatístico obtido na classificação horizontal conforme ISO 17.123-3 - teste completo T105.

\begin{tabular}{|c|c|}
\hline \multicolumn{2}{|c|}{ TESTE ESTATÍSTICO DA CLASSIFICAÇÃO DO INSTRUMENTO (ÂNGULOS HORIZONTAIS } \\
\hline \multicolumn{2}{|c|}{ "O desvio-padrão experimental $S$, é menorifigual ao desvio padrão, $S f$, fornecido pelo fabricante" } \\
\hline Desvio padrão experimental & Desvio padrão do fabricante \\
\hline calculado S (") & Sf (") \\
\hline 8,00 & 10 \\
\hline \multicolumn{2}{|c|}{ "HIPÓTESE NULA ACEITA - RESULTA OK - S<Sf } \\
\hline \multicolumn{2}{|c|}{$\begin{array}{l}\text { "Considerando dois desvios padrão experimentais calculados, sendo: } \\
\text { S o desvio padrão experimental calculado na classificação e } \\
\text { Smenor, o menor desvio padrão experimental calculado entre as } 4 \text { séries. } \\
\text { Estes dois desvios padrão pertencem a mesma população?" }\end{array}$} \\
\hline Desvio padrão experimental & Menor desvio padrão calculado \\
\hline calculado S (") & nas 4 séries: Smenor (") \\
\hline Desv. Padrão série 2 (S2) (") & 3,6514 \\
\hline "HIPĆ & CEITA" \\
\hline
\end{tabular}

Os testes de classificação angular vertical utilizaram os teodolitos eletrônicos T105 (precisão \pm 5 ") e T100 (precisão \pm 10 ") e estação total TC403 L (precisão \pm 10 "). Para procedimento em laboratório o sistema de ar-condicionado é acionado do início do teste, mantendo uma temperatura média entre $21^{\circ} \mathrm{C}$ e $22^{\circ} \mathrm{C}$. A umidade relativa do ar no laboratório também foi monitorada permanecendo em torno de $61 \%$. Para a classificação angular vertical são realizadas 4 séries completas de PD e PI, em 3 conjuntos, utilizando 4 colimadores, de acordo com o estabelecido pela ISO 17.123-3. Devido ao volume de dados, ressalta-se a importância da atenção na pontaria, anotação e seqüência das leituras para a finalização da classificação com êxito.

\subsection{Comparação entre Bases de Campo, Laboratório com Colimadores Construídos e Laboratório com Colimadores Leica}

Para a consolidação dos resultados da base de classificação angular de laboratório utilizando colimadores construídos, se fez necessário confrontar os resultados obtidos anteriormente com os resultados obtidos pelo sistema colimador Leica. Para isso, foram realizados 5 testes para a classificação horizontal e 5 testes para a classificação vertical, sendo que duas vezes com o mesmo equipamento em cada classificação, para garantir a repetibilidade dos resultados.

Os equipamentos testados foram teodolitos eletrônicos T100, T105 (duas vezes) e a estação total TC403 L. O procedimento de execução dos testes de classificação foi similar ao realizado nos colimadores construídos. $\mathrm{O}$ instrumento foi posicionado sobre o pilar central da base de classificação e na seqüência, iniciou-se o procedimento de obtenção das observações. $\mathrm{O}$ teste completo para 
classificação angular horizontal totaliza 120 observações e 96 observações para a classificação angular vertical.

\section{ANÁLISE E DISCUSSÃO DOS RESULTADOS}

Os testes realizados nas bases de classificação horizontal de campo e laboratório permitiram realizar a comparação dos resultados obtidos com bases para validar a proposta da pesquisa.

O primeiro teste apresentado refere-se ao teodolito T105 Leica, com precisão nominal de \pm 5 ". Em campo foi realizado um teste seguindo todos os cuidados citados anteriormente. Em laboratório foram realizadas 2 classificações com 4 séries de observações completas cada. Uma vez com o colimador construído e duas vezes com o colimador Leica, feitas em dois dias diferentes para que a habilidade e acuidade do observador não interferissem no resultado. $\mathrm{O}$ quadro 2 apresenta os valores obtidos nas classificações feitas em campo e laboratório.

Quadro 2 - Resultados dos testes de classificação e estatísticos da classificação horizontal da base de laboratório e campo com teodolito T105 N5.

\begin{tabular}{|c|c|c|c|c|}
\hline Equipamento & $\begin{array}{c}\text { Precisão no } \\
\text { laboratório }\end{array}$ & Classificação & $\begin{array}{c}\text { Hipótese nula } \\
\left(\sigma_{\text {exp }} \leq \sigma_{\text {fab }}\right)\end{array}$ & $\begin{array}{c}\text { Hipótese nula } \\
\text { (mesma } \\
\text { população) }\end{array}$ \\
\hline $\begin{array}{c}\text { Colimador } \\
\text { Leica (23/05) }\end{array}$ & $4,14 ”$ & Média & Aceita & Aceita \\
\hline $\begin{array}{c}\text { Colimador } \\
\text { Leica (24/05) }\end{array}$ & $3,33 ”$ & Média & Aceita & Aceita \\
\hline $\begin{array}{c}\text { Colimador } \\
\text { Construído }\end{array}$ & $4,04 ”$ & Média & Aceita & Aceita \\
\hline $\begin{array}{c}\text { Base de } \\
\text { campo }\end{array}$ & $4,97 ”$ & Média & Sem avaliação & Sem avaliação \\
\hline
\end{tabular}

De acordo com os resultados obtidos, observa-se que os dados da base de laboratório apresentaram-se próximos a precisão nominal do equipamento em teste $( \pm 5$ "), sendo estes os desvios-padrão obtidos em laboratório $( \pm 4,14$ ", $\pm 3,33$ " e $\pm 4,04$ "), e o obtido na base de campo $( \pm 4,97)$.

Após a confirmação da eficiência da base de laboratório, realizaram-se 4 classificações angulares completas para a componente horizontal. Foram aplicados os testes nos seguintes equipamentos: 2 teodolitos eletrônicos T105, 1 teodolito eletrônico T100 e uma estação total TC403L. Para efeitos de comparação realizouse a classificação destes equipamentos também na base de campo. O quadro 3 apresenta os valores de precisão e classificação horizontal de cada equipamento, em ambas as bases. 
Quadro 3 - Resultados da classificação horizontal da base de campo e base de laboratório.

\begin{tabular}{|l|c|c|c|c|c|}
\hline Equipamento & $\begin{array}{c}\text { Precisão } \\
\text { nominal }\end{array}$ & $\begin{array}{c}\text { Resultado } \\
\text { campo }\end{array}$ & $\begin{array}{c}\text { Classificação } \\
\text { campo }\end{array}$ & $\begin{array}{c}\text { Resultado } \\
\text { laboratório }\end{array}$ & $\begin{array}{c}\text { Classificação } \\
\text { laboratório }\end{array}$ \\
\hline Teo. 105 n5 & $\pm 5 "$ & $\pm 4,97 ”$ & Média & $\pm 4,04 ”$ & Média \\
\hline Teo. 105 n4 & $\pm 5 "$ & $\pm 3,28 ”$ & Média & $\pm 3,01 ”$ & Média \\
\hline Teo.T100 n7 & $\pm 10 ”$ & $\pm 6,24 ”$ & Média & $\pm 4,51 ”$ & Média \\
\hline ET TC403L & $\pm 10 "$ & $\pm 3,25 "$ & Média & $\pm 4,15 ”$ & Média \\
\hline
\end{tabular}

$\mathrm{Na}$ comparação dos resultados verifica-se que para todos os equipamentos testados existiu compatibilidade dos valores de precisão e a mesma classificação obtida no teste de campo repetiu-se no laboratório.

Para avaliar os resultados obtidos em laboratório, estes foram submetidos à avaliação empregando-se testes estatísticos. O primeiro teste estatístico analisou o desvio-padrão experimental ( $\sigma$ exp) obtido em função do desvio-padrão fornecido pelo fabricante ( $\sigma \_$fab). Neste caso a hipótese nula do teste é aceita caso o desviopadrão calculado seja menor ou igual ao fornecido pelo fabricante. O segundo teste avalia a compatibilidade das amostras (observações). Para esta situação a hipótese nula é aceita caso o desvio-padrão calculado na classificação e o menor desviopadrão obtido entre as séries de observações pertencem a mesma população, o quadro 4 mostra os resultados destes testes.

Quadro 4 - Resultados dos testes estatísticos da classificação horizontal da base de laboratório.

\begin{tabular}{|l|c|c|c|}
\hline Equipamento & $\begin{array}{c}\text { Precisão no } \\
\text { teste } \\
\text { de laboratório }\end{array}$ & $\begin{array}{c}\text { Hipótese nula } \\
\left(\sigma_{-} \text {exp } \leq \sigma \_f a b\right)\end{array}$ & $\begin{array}{c}\text { Hipótese nula } \\
\text { (mesma } \\
\text { população) }\end{array}$ \\
\hline Teo 105 n5 & $\pm 4,04 "$ & Aceita & Aceita \\
\hline Teo 105 n4 & $\pm 3,01 "$ & Aceita & Aceita \\
\hline Teo T100 n7 & $\pm 4,51 "$ & Aceita & Aceita \\
\hline ET TC403L & $\pm 4,15 "$ & Aceita & Aceita \\
\hline
\end{tabular}

Os testes estatísticos comprovam a adequação das observações e a confiabilidade do resultado. Ambas as questões acerca do desvio-padrão calculado foram aceitas. Ressalta-se que os testes estatísticos não foram aplicados aos resultados da base de campo, pois a norma NBR 13.133, que estabelece os critérios para a classificação angular em campo, não prevê esta situação.

Feita a experimentação para a base de classificação angular horizontal, partiuse para a classificação angular vertical. Considerando os resultados obtidos na classificação horizontal em laboratório, os mesmos parâmetros foram considerados 
para a classificação vertical. Isto foi adotado devido inexistência de uma normativa brasileira que contemple a questão angular vertical. Desta forma, os testes de classificação foram baseados na ISO17.123-3 e a classificação em função do desvio-padrão experimental obtido em função da NBR 13.133.

Foram utilizados nestes testes os seguintes equipamentos: 2 teodolitos eletrônicos T105, 1 teodolito eletrônico T100 e uma estação total TC403L. No quadro 5 são apresentados os resultados obtidos.

Quadro 5 - Resultados da classificação angular vertical da base de laboratório.

\begin{tabular}{|l|c|c|c|}
\hline Equipamento & $\begin{array}{c}\text { Precisão } \\
\text { nominal }\end{array}$ & $\begin{array}{c}\text { Precisão nos } \\
\text { testes } \\
\text { de laboratório }\end{array}$ & Classificação \\
\hline Teo 105 n5 & $\pm 5 "$ & $\pm 2,92 ”$ & Média \\
\hline Teo 105 n4 & $\pm 5 "$ & $\pm 3,52 "$ & Média \\
\hline Teo T100 n7 & $\pm 10 "$ & $\pm 4,79 "$ & Média \\
\hline ET TC403L & $\pm 10 "$ & $\pm 4,38 ”$ & Média \\
\hline
\end{tabular}

Observando-se os resultados verifica-se que para todos os equipamentos testados existiu compatibilidade dos valores de precisão nominal (ou informada pelo fabricante) e o valor de precisão obtida no teste. Estes resultados apoiados pelos resultados da classificação horizontal reafirmam o êxito na implantação do laboratório. Salienta-se que de acordo com os resultados obtidos para os equipamentos T100 e TC403L, ambos foram classificados em laboratório como de média precisão na classificação angular vertical (os quais seriam classificados de baixa precisão, de acordo com sua precisão nominal).

A classificação vertical também conta com apoio da avaliação dos parâmetros estatísticos. O quadro 6 mostra os resultados destes testes.

Quadro 6 - Resultados dos testes estatísticos da classificação vertical base de laboratório.

\begin{tabular}{|l|c|c|c|c|}
\hline Equipamento & $\begin{array}{c}\text { Precisão no } \\
\text { laboratório }\end{array}$ & $\begin{array}{c}\text { Hipótese nula } \\
\left(\sigma_{-} \text {exp } \leq \sigma_{-} \text {fab) }\right.\end{array}$ & $\begin{array}{c}\text { Hipótese nula } \\
\text { (mesma } \\
\text { população) }\end{array}$ & $\begin{array}{c}\text { Hipótese } \\
\text { nula } \\
\text { (não há erro } \\
\text { de índice } \\
\text { vertical) }\end{array}$ \\
\hline Teo 105 n5 & $\pm 2,92 ”$ & Aceita & Aceita & Rejeitada \\
\hline Teo 105 n4 & $\pm 3,52 ”$ & Aceita & Aceita & Rejeitada \\
\hline Teo T100 & $\pm 4,79 ”$ & Aceita & Aceita & Aceita \\
\hline ET TC403L & $\pm 4,38 ”$ & Aceita & Aceita & Aceita \\
\hline
\end{tabular}

Os testes estatísticos comprovam a adequação das observações e a confiabilidade do resultado. Ambas as questões acerca do desvio-padrão calculado 
foram aceitas. Em virtude dos resultados apresentados, cabe a verificação dos teodolitos eletrônicos T105 números 4 e 5, sobre a questão do erro de índice vertical, pois ambos foram rejeitados com relação a este teste.

Para a verificação final na base de laboratório, realizaram-se testes com os equipamentos utilizados em campo e laboratório com os colimadores construídos, nos testes com os colimadores industriais Leica. O teodolito eletrônico T105 No5 foi submetido a duas classificações, uma no dia 23 e outra no dia 24 . Este procedimento foi adotado para verificar a repetibilidade das observações em situações distintas.

O quadro 7 mostra os resultados alcançados para a classificação angular horizontal para o teodolito T105 $\mathrm{N}^{0} 5$, no primeiro teste $(23 / 05)$, segundo teste (24/05), bem como os resultados obtidos no laboratório com colimador Leica, colimador construído e base de campo.

Os resultados obtidos são compatíveis com a precisão nominal do equipamento que é de \pm 5 ”, portanto classificando-o como de média precisão. Pelos dados da tabela pôde-se observar que todos os procedimentos utilizados chegaram a mesma classificação do equipamento, sendo importante ressaltar o desempenho do colimador construído no laboratório, cujos resultados ficaram muito próximos dos apresentados pela base de campo da UFPR e pelo colimador industrial.

Quadro 7 - Resultados dos testes de classificação e estatísticos da classificação angular horizontal base de laboratório.

\begin{tabular}{|l|c|c|c|c|}
\hline Equipamento & $\begin{array}{c}\text { Precisão no } \\
\text { laboratório }\end{array}$ & Classificação & $\begin{array}{c}\text { Hipótese nula } \\
\left(d p_{\text {_exp }} \leq d p_{\text {_fab }}\right)\end{array}$ & $\begin{array}{c}\text { Hipótese nula } \\
\text { (mesma } \\
\text { população }\end{array}$ \\
\hline $\begin{array}{l}\text { T105 n5 } \\
(23 / 05 / 08) \\
\text { Col. Leica }\end{array}$ & $\pm 4,14 ”$ & Média & Aceita & Aceita \\
\hline $\begin{array}{l}\text { T105 n5 } \\
(24 / 05 / 08) \\
\text { Col. Leica }\end{array}$ & $\pm 3,33 ”$ & Média & Aceita & Aceita \\
\hline $\begin{array}{l}\text { Colimador } \\
\text { Construído }\end{array}$ & $\pm 4,04 ”$ & Média & Aceita & Aceita \\
\hline $\begin{array}{l}\text { Base de } \\
\text { campo }\end{array}$ & $\pm 4,97 ”$ & Média & Sem avaliação & $\begin{array}{c}\text { Sem } \\
\text { avaliação }\end{array}$ \\
\hline
\end{tabular}

O quadro 8 mostra os resultados alcançados para a classificação angular vertical para o teodolito T105 $\mathrm{N}^{\circ} 5$, no primeiro teste (23/05) e segundo teste (24/05) com os colimadores industriais e com o colimador construído. 
Quadro 8 - Resultados dos testes de classificação e estatísticos da classificação vertical da base de laboratório.

\begin{tabular}{|c|c|c|c|c|c|}
\hline $\begin{array}{c}\text { Equipament } \\
\text { o }\end{array}$ & $\begin{array}{c}\text { Precisão no } \\
\text { laboratório }\end{array}$ & $\begin{array}{c}\text { Classifi- } \\
\text { cação }\end{array}$ & $\begin{array}{c}\text { Hipótese nula } \\
\left(\sigma_{-} \exp \leq \sigma_{-} f a b\right.\end{array}$ & $\begin{array}{c}\text { Hipótes } \\
\text { e nula } \\
\text { (mesma } \\
\text { pop.) }\end{array}$ & $\begin{array}{c}\text { Hipótese nula } \\
\text { (não há erro de } \\
\text { indice vertical) }\end{array}$ \\
\hline $\begin{array}{c}\text { T105n5 } \\
(23 / 05 / 08)\end{array}$ & $\pm 4,73 ”$ & Média & Aceita & Aceita & Aceita \\
\hline $\begin{array}{c}\text { T105n5 } \\
(24 / 05 / 08)\end{array}$ & $\pm 2,37 ”$ & Média & Aceita & Aceita & Aceita \\
\hline $\begin{array}{c}\text { Colimador } \\
\text { Construído }\end{array}$ & $\pm 2,92 ”$ & Média & Aceita & Aceita & Aceita \\
\hline
\end{tabular}

Os resultados apresentados no quadro 8 estão abaixo da precisão nominal do equipamento que é de \pm 5 ", portanto classificando-o como de média precisão. Portanto, pôde-se observar que todos os procedimentos utilizados chegaram à mesma classificação do equipamento, sendo importante ressaltar o desempenho do colimador construído no laboratório.

De uma forma geral, comparando-se o tempo para a obtenção dos testes de campo e de laboratório, o procedimento de laboratório mostrou-se $64 \%$ mais rápido e $66 \%$ mais econômico em relação ao número de pessoas envolvidas, já que em campo são necessárias três pessoas para realização do teste e em laboratório apenas uma.

\section{CONSIDERAÇÕES FINAIS}

Os estudos e experimentos desenvolvidos neste trabalho visaram atender as necessidades da comunidade usuária de equipamentos de medição angular, com relação à classificação angular horizontal e vertical. A classificação horizontal foi apoiada pela base de campo e por estudos realizados com a ISO 17.123-3. Neste caso os equipamentos foram submetidos a testes de campo e também na base de laboratório construída com colimadores desenvolvidos no laboratório. Portanto, foi possível a comparação dos resultados obtidos em ambas as classificações e a partir desses resultados validar a base de laboratório, que é constituída por colimadores construídos e por colimadores industriais (marca Leica). Para esta validação realizaram-se testes nas três bases sugeridas e os resultados obtidos consolidam a proposta da metodologia empregada, pois a partir de lunetas de equipamentos com avarias mecânicas, pôde-se construir um colimador eficiente e de baixo custo que forneceu resultados compatíveis com os colimadores adquiridos.

O procedimento de classificação executado no laboratório também se mostrou eficiente em comparação ao realizado em campo. No laboratório o desgaste do operador é menor em relação ao ambiente de campo. A climatização do ambiente 
propicia minimização dos efeitos ambientais, além do conforto para tomada das observações.

Nos testes realizados na base de laboratório para classificação angular horizontal, demonstrou-se através dos experimentos com as diferentes iluminações, que este fator pode influenciar na focalização dos retículos e por conseqüência embutir erros na classificação. Com relação à base de classificação angular vertical, foram necessários mais alguns estudos, pois não existia a possibilidade de comparações como no caso da classificação angular horizontal. Devido a inexistência de procedimentos de classificação angular vertical em normas brasileiras, admitiram-se então os mesmos valores e classificação sugeridos pela NBR 13.133 para a classificação angular horizontal.

Frente a essas considerações pode-se concluir que o objetivo inicial da proposta de pesquisa foi plenamente atingindo, sendo ao final disponibilizado à comunidade um laboratório para classificação angular horizontal e vertical de instrumentos de medição angular.

\section{AGRADECIMENTOS}

Os autores gostariam de agradecer a Fundação Araucária de Apoio ao Desenvolvimento Científico e Tecnológico do Paraná pelo apoio ao projeto de pesquisa intitulado "Implantação de laboratório para verificação, retificação e classificação da componente angular de teodolitos e estações totais em ambientes controlados ", na aquisição dos colimadores.

\section{REFERÊNCIAS BIBLIOGRÁFICAS}

ISO 17.123-3: Óptics and optical instruments - Field procedures for testing geodetic and surveying instruments - Part 3: Theodolites. Switzerland, 2002.

MEDINA, A. S. Classificação de teodolitos e estações totais na UFPR. Curitiba; 1998; 108 f. Dissertação (Mestrado em Ciências Geodésicas) - Universidade Federal do Paraná, Curitiba.

NBR 13.133: Execução de levantamento topográfico. Rio de Janeiro: ABNT, 1994.

Recebido em maio de 2009. Aceito em junho de 2010. 